\title{
Organisations islamiques en Europe et aux États-Unis: les perspectives interdisciplinaires et ascendantes
}

2015, Vol. 62(4) 54I-555

(C) The Author(s) 2015

Reprints and permissions: sagepub.co.uk/journalsPermissions.nav DOI: $10.1177 / 0037768615602193$

scp.sagepub.com

\section{Matthias KORTMANN}

Ludwig-Maximilians-Universität München, Allemagne

\section{Kerstin ROSENOW-WILLIAMS}

Ruhr-Universität Bochum, Allemagne

\section{Résumé}

Cet article utilise une structure analytique qui examine les différences concernant les formes organisationnelles et les stratégies des organisations islamiques $(\mathrm{OI})$ prenant pour sources à la fois les facteurs internes et externes ayant un effet sur l'organisation, tels que les caractéristiques d'organisations internes ou les structures d'opportunités politiques nationales et transnationales. Cette perspective est appliquée pour réexaminer les résultats empiriques d'études secondaires prenant une perspective nationale et transatlantique. Plus particulièrement, ce texte se centre sur une méta-analyse des données obtenues après une recherche dans l'ouvrage Islamic organizations in Europe and the USA: A multidisciplinary perspective, édité par Kortmann et Rosenow-Williams (20I3), qui présente de multiples recherches empiriques sur les $\mathrm{Ol}$ aux États-Unis, en Allemagne, en France, aux Pays-Bas, en Angleterre, dans les pays baltes, en Pologne, en Espagne, en Belgique et en Suisse.

\section{Mots-clés}

États-Unis, Europe, intégration, islam, organisation

\footnotetext{
Pour toute correspondance :

Matthias Kortmann, Department of Political Science, Ludwig-Maximilians-Universität München, Oettingenstr. 67, München 80538, Allemagne

Email : Matthias.Kortmann@gsi.uni-muenchen.de
} 


\begin{abstract}
This article utilizes an analytical framework that examines the differences in the organizational forms and strategies of Islamic organizations with reference to both internal and external factors affecting the organization, such as internal organizational characteristics and national and transnational political opportunity structures. This perspective is applied to review the empirical results of recent secondary studies from a cross-national and transatlantic perspective. In particular, this paper centres around a meta-analysis of research findings collected in the volume Islamic organizations in Europe and the USA: A multidisciplinary perspective, edited by Kortmann and Rosenow-Williams (20I3), which presents new empirical research on Islamic organizations in the USA, Germany, France, the Netherlands, the UK, the Baltic States, Poland, Spain, Belgium and Switzerland.
\end{abstract}

\title{
Keywords
}

Europe, integration, Islam, organization, USA

\section{Introduction}

Depuis le début du $21^{\mathrm{e}}$ siècle, les questions autour de l'intégration des musulmans dans la société, et les apports de leurs organisations dans les relations clergé-État dans des pays n'étant pas à majorité musulmane, ont été largement discutées par des universitaires et des politiciens à la fois en Europe et aux États-Unis. Ce problème a amené de nombreuses études basées sur une perspective descendante concernant les traitements des États envers les minorités musulmanes et leurs organisations dans le contexte de différents régimes nationaux de gouvernance religieuse et de diversité religieuse (e.g. Bader, 2007; Cesari, 2009; Koenig, 2007; Laurence, 2012 ; Nielsen et al., 2012).

Le but de cet article est de démontrer l'utilité d'une approche ascendante dans l'analyse des organisations islamiques (OI) et les enjeux que ces organisations rencontrent dans les deux régions concernées dans cette recherche : l'Europe et les États-Unis ${ }^{1}$. L'approche ascendante se focalise sur les perspectives d'acteurs sociaux précis dans le champ politique en fonction de leurs intérêts spécifiques. Ici, les OI et leurs membres sont les principales unités d'analyse. Cette approche comprend une étude de leur environnement, leurs discours public et politique, leurs stratégies de réponse et leurs formes organisationnelles. Un tel angle de recherche donne de multiples avantages. Premièrement, il élargit notre compréhension de la façon dont les musulmans «organisent» leurs intérêts et leur religion en générale. Deuxièmement, il permet de donner un aperçu de la façon dont cette «gestion» diffère selon les différents contextes nationaux, les acteurs collectifs et les groupes ou les «courants» à l'intérieur de l'islam elle-même. Troisièmement, cette approche englobe non seulement des micro-analyses de l'action sociale des individus et des dirigeants des OI, mais aussi des recherches au niveau méso de la façon dont les OI interagissent avec leurs membres et les contextes sociaux. 
Le cadre analytique de cet article examine les différences de formes organisationnelles et de stratégies des OI en référence aux facteurs internes et externes qui les influencent, tels que les caractéristiques organisationnelles internes ou les structures d'opportunités politiques nationales et transnationales. Cette perspective est ensuite appliquée pour examiner les résultats empiriques de récentes études secondaires prenant une perspective nationale et transatlantique.

Cet article repose sur une méta-analyse de résultats de recherche collectés dans l'ouvrage Islamic organizations in Europe and the USA: A multidisciplinary perspective, édité par Kortmann et Rosenow-Williams (2013), qui présente plusieurs recherches empiriques sur les OI aux États-Unis et dans quelques pays d'Europe. Des études additionnelles, qui sont basées sur de récentes recherches empiriques, sont aussi incorporées dans la méta-analyse pour donner une perspective plus large sur ce domaine. Les études analysées incluent toute une variété de méthodologies appliquées par des auteurs venant de multiples disciplines de recherche, incluant la sociologie organisationnelle, les sciences politiques, la théologie, les études de l'islam et l'anthropologie.

\section{Questions de recherche et cadre théorique}

Les études en général démontrent que l'hétérogénéité est une caractéristique fondamentale des OI en Europe, aux États-Unis, et dans d'autres parties du monde. Cette analyse examine l'hétérogénéité organisationnelle des OI selon trois niveaux: (1) l'impact du statut de minorité sur les formes d'organisation qui fleurissent ou non dans les contextes de minorités musulmanes; (2) l'impact des divers liens transnationaux entretenus par les OI sur leurs formes organisationnelles; et (3) les dynamiques organisationnelles internes aux niveaux des organisations des mosquées locales.

Le premier niveau d'analyse évalue les formes organisationnelles dominantes parmi les OI dans les pays sélectionnés. Les questions fondamentales abordées sont les suivantes : quelles stratégies les OI adoptent-elles aux niveaux nationaux, régionaux et locaux envers leur environnement organisationnel interne et externe? Comment leurs comportements sont-ils façonnés par leur reconnaissance, ou statut organisationnel, à chaque niveau? Le cadre de recherche sous-jacent intègre l'analyse des facteurs contextuels externes mis en évidence par le concept de «structures d'opportunité politique» (SOP), souvent utilisé pour expliquer les tendances dans la participation politique des organisations de migrants. Koopmans et Statham (2000) proposent à la fois des facteurs formels, tel que le contexte institutionnel national, et des facteurs informels, telle que la culture politique d'une société, comme variables explicatives des possibilités et des limites dans la mobilisation politique des migrants. "L'ensemble des SOP pertinentes pour l'intégration politico-culturelle des minorités ethniques $»^{2}$ (Duyvené de Wit et Koopmans, 2005 : 52) se compose des droits individuels et des droits collectifs des groupes. Enfin, Koopmans et al. (2005: 19) se réfèrent également aux opportunités discursives qui définissent «quelles identités collectives et exigences de fond ont une forte probabilité de gagner en visibilité dans les médias de masse, en résonant avec les revendications des autres acteurs collectifs et d'atteindre une légitimité dans le discours public». 
Ces dernières années, les approches SOP ont été étendues pour inclure les ambitions politiques des groupes minoritaires islamiques qui ont pour revendications des décisions qui reflètent les orientations normatives de la gouvernance religieuse de leurs Étatsnations respectifs. Par exemple, dans leur étude sur les musulmans et l'État au RoyaumeUni, en France et en Allemagne, Fetzer et Soper (2005) examinent les opportunités des «nouvelles» religions comme l'islam de participer aux relations église-État au niveau national. Plusieurs études se sont penchées sur le lien entre les modèles nationaux de gouvernance religieuse et l'intégration des musulmans dans les pays européens, mettant en valeur les différences nationales dans les modèles de gouvernance religieuse et les manières dont ils affectent les opportunités d'intégration des communautés religieuses islamiques (pour l'Europe, voir Bader, 2007; Koenig, 2007; Statham et al., 2009).

Les possibilités institutionnelles et discursives varient en fonction de leur accueil dans les OI. Les régimes multiculturels d'intégration des immigrants sont généralement plus ouverts que les régimes assimilationnistes. Il en va de même pour les régimes de gouvernance religieuse qui accentuent le pluralisme religieux en comparaison avec les régimes qui se caractérisent par la présence d'une église établie. Un contexte fermé envers les migrants ou les musulmans se traduira par des opportunités discursives plus limitées que dans les sociétés qui sont plus ouvertes au dialogue avec les «nouveaux arrivants». Enfin, les SOP peuvent changer avec le temps, à la suite de réformes structurelles (influençant les opportunités institutionnelles) ou à la suite d'événements soudains largement débattus (impactant les opportunités discursives).

Le deuxième niveau d'analyse examine la façon dont les OI sont influencées par les liens transnationaux qu'elles entretiennent avec des acteurs étrangers. Le cadre de recherche sous-jacent est fondé sur l'approche de recherche "transnationaliste», qui, depuis les années 1990, est devenu un concept largement utilisé dans les sciences sociales pour l'analyse de phénomènes en rapport avec la migration qui s'étendent au-delà des frontières territoriales. Cet axe de recherche se concentre sur le caractère transnational des organisations de migrants et de la vie des migrants qui «entretiennent des relations sociales multiples qui font le lien entre leurs sociétés d'origines et celles où ils vivent» (Basch et al., 1997: 7). Basch et al. (1997), entre autres, mettent en évidence la nécessité d'aller au-delà du piège du «nationalisme méthodologique», qui s'exprime dans des plans de recherche se limitant à des phénomènes sociaux à l'intérieur des frontières existantes des États-nations. La littérature sur le transnationalisme indique donc que les réseaux individuels et organisationnels, ayant des dimensions culturels, économiques, politiques ou religieuses, s'étendent sur deux ou plusieurs espaces géographiques (Østergaard-Nielsen, 2003; Pries et Sezgin, 2012; Sunier et Landman, 2015). Dans notre méta-analyse, nous analysons particulièrement comment les OI utilisent stratégiquement leurs liens multiples tant dans les pays à majorité islamique que dans les pays à minorité islamique pour répondre aux environnements organisationnels de leurs sociétés «hôtes».

Le troisième niveau d'analyse présenté ici se concentre sur les dynamiques organisationnelles internes des mosquées locales. Les dirigeants des OI tels les imams, mais aussi les porte-paroles ou les représentants élus, doivent également répondre aux attentes de la société en général, mais aussi satisfaire les besoins des membres de l'organisation (Rosenow-Williams, 2012). Schmitter et Streeck (1999) illustrent ce 
dilemme des organisations en contrastant les logiques de l'adhésion et de l'influence. D'après la logique de l'adhésion, une organisation dépend de ses membres à la fois comme une ressource mais aussi pour sa survie. Parallèlement, la logique d'influence implique que l'organisation doit exercer une influence sur les autorités publiques et obtenir des ressources de leur part sous forme de reconnaissance, de légitimité ou d'un statut officiel (Schmitter et Streeck, 1999: 19).

La recherche examinée à partir de ce niveau d'analyse se concentre sur les stratégies organisationnelles et les pratiques religieuses qui varient selon les contextes nationaux et entre les différents groupes religieux ou entre les différentes formes organisationnelles de l'islam lui-même. La réaction des communautés locales «hôtes» à la présence d'OI peut être une source d'hétérogénéité à ce niveau, comme peuvent l'être les attitudes divergentes face à l'intégration des membres et des directeurs organisationnels eux-mêmes.

Ce troisième niveau d'analyse présente un autre point d'intérêt comparatif important concernant l'impact de la variation de régime politique interne (par exemple: directeurs élus ou imams nommés) sur le comportement organisationnel vis-à-vis de la communauté locale et de l'État. Certaines organisations fonctionnent comme des communautés avec une approche participative de la prise de décision et une structure ascendante tandis que d'autres sont hiérarchiquement structurées et ne reposent pas sur un sentiment de communauté parmi les membres (cf. Gest, 2013; Malik, 2013 ; Nalborczyk et Ryszewska, 2013).

Enfin, il existe des différences entre les mosquées dans la mesure où celles-ci fournissent des services spécifiques pour garder et attirer des membres, tels que des aides aux démarches administratives ou juridiques, la scolarité, l'organisation des rituels religieux (comme le pèlerinage Hajj à la Mecque) ou les contacts avec les autorités locales. Ces services diffèrent selon les contextes nationaux en raison de différents règlements institutionnels des groupes religieux dans chaque pays, et ils diffèrent également entre les groupes islamiques dans un seul État en raison des variations des ressources organisationnelles, du niveau d'acceptation au sein des communautés locales, de leur identité religieuse et culturelle, et des attentes internes des membres de leur mosquée.

\section{L'état des recherches en approche ascendante sur les Ol : une évaluation systématique}

Quand nous observons la communauté des OI, nous réalisons rapidement que «l'islam» ou «l'OI» n'existe pas en tant que telle dans n'importe quel État-nation. Cela peut être attribué aux formes, stratégies et pratiques hétérogènes des OI à différents niveaux et dans différents endroits. Les résultats de cette méta-analyse permettent de mieux comprendre les diverses façons qu'ont les musulmans de participer à la politique et d'exercer leur foi dans les pays à majorité non-musulmane.

L'analyse qui suit se concentre principalement sur l'identification de plusieurs facteurs externes contextuels qui expliquent l'émergence de différences entre les OI dans les différents pays d'Europe et d'Amérique du Nord, en prenant les OI pour unité d'analyse. 


\section{L'impact de l'hétérogénéité contextuelle sur la structure organisationnelle}

Trois facteurs sont apparus de la méta-analyse des études comparatives récentes examinées ici : (a) les $S O P$ qui émergent non seulement des règles et règlements qui façonnent la gouvernance religieuse, mais aussi des structures politiques et économiques au niveau de l'État, (b) les opportunités discursives à différents niveaux de l'action sociale, et (c) les différents régimes d'incorporation des immigrants dans différents contextes nationaux, et les liens transnationaux des OI, qui peuvent paraître à première vue comme des facteurs distincts, mais sont en fait liés. Les nombreuses influences de ces divers facteurs contextuels présents du niveau supranational au niveau local ne concernent pas seulement les processus de construction de l'organisation, mais aussi la façon dont ces organisations établissent des programmes internes et externes, comme souligné ci-dessous.

Facteur contextuel I : les SOP. Dans une étude de cas illustrant l'impact du régime national de la gouvernance religieuse, la recherche de Nalborczyk et Ryszewska (2013) sur les organisations musulmanes polonaises démontre que les structures des OI augmentent en pluralité en raison d'un contexte juridique évolutif qui a accordé une reconnaissance légale à un nombre croissant d'OI, augmentant ainsi la concurrence dans le domaine de l'organisation. Les auteurs montrent que, dans leurs efforts pour être reconnues comme des communautés religieuses, les OI cherchent à transformer leurs structures se conformant aux critères établis par le système juridique polonais.

Ainsi, dans son analyse des OI dans les trois États baltes, Račius (2013) soutient que les structures organisationnelles islamiques sont influencées par des régimes nationaux divergents de gouvernance religieuse. En Lituanie, l'islam sunnite est reconnue comme l'une des religions « traditionnelles» du pays, tandis qu'en Estonie et en Lettonie, l'islam a un statut moins favorable car elle est perçue comme «non-traditionnelle». Leur statut «traditionnel» fournit aux OI en Lituanie des ressources juridiques dans leurs efforts pour établir, par exemple, des cimetières islamiques ou pour introduire l'éducation religieuse, tandis que leurs homologues en Estonie et en Lettonie ont dû surmonter des obstacles plus importants. Račius traite des diverses stratégies que les OI élaborent en réponse aux cadres juridiques et aux environnements sociaux qui peuvent être nocifs, voire hostiles aux musulmans. Cette hostilité se nourrit de la crainte que la présence musulmane ne change les coutumes et les pratiques traditionnelles nationales et locales. Pour faire face à une telle situation, les groupes s'abstiennent parfois de faire des déclarations publiques ou des demandes, dans l'espoir qu'ils seront «laissés en paix à la fois par l'État et la société» (Račius, 2013: 127).

Dans son étude comparative des organisations de migrants en Allemagne et aux PaysBas, Kortmann (2011) utilise le concept de SOP afin d'expliquer non seulement comment les musulmans sont mobilisés politiquement, comment ils établissent des organisations, mais aussi comment ils élaborent des stratégies qu'ils présentent dans l'arène politique. En Allemagne, certaines options en faveur des organisations religieuses dans la Loi fondamentale $(\mathrm{GG})$ offrent des possibilités pour les OI d'être reconnues à la fois comme communautés religieuses - ayant par conséquent la capacité de délivrer des cours de 
religion islamique dans les écoles publiques (Art. $7 \mathrm{GG}$ ) - et comme corporations religieuses, selon le droit public (Art. $140 \mathrm{GG}$ ). Les OI en Allemagne ont longtemps essayé d'obtenir le statut de «corporation religieuse», car cela leur donne droit à certains privilèges de droit public, tel que le droit de percevoir une «taxe de l'église» par leurs membres ou d'être invitées aux conseils consultatifs gouvernementaux. D'autre part, les OI aux Pays-Bas, où il n'existe aucune possibilité comparable pour les organisations religieuses, ont tendance à minimiser leur caractère religieux, se dépeignant plutôt comme des organisations socio-culturelles dans l'espoir d'un financement public.

Alors que les OI en Europe se présentent principalement comme des homologues religieux des églises chrétiennes et exigent les mêmes droits et privilèges prévus par les régimes nationaux de gouvernance religieuse, les OI aux États-Unis utilisent une stratégie différente. Elles ne se définissent pas prioritairement comme des acteurs religieux dans leurs efforts pour obtenir l'égalité des droits. Au contraire, Love (2013) trouve des parallèles avec les stratégies appliquées par les organisations de défense des droits des « communautés de minorités racialisées». Celles-ci en sont venues récemment à préférer le mode de défense des libertés civiles «color-blind» au mode des groupes des droits civiques qui ont traditionnellement considéré des attributs tels que la «race». Ce mode de libertés civiles «souligne la perspective que tous les citoyens méritent un traitement égal, sans distinction de race, de sexe, de religion, ou d'autres caractéristiques d'identité» (Love, 2013 : 41). Quand Azzaoui (2009: 3) compare les OI en Allemagne et aux ÉtatsUnis, ses résultats confirment cette différence d'image de soi des OI dans le contexte américain et européen. Alors que les OI en Allemagne «concentrent leur travail principalement sur des sujets religieux et politiques qui les concernent», comme les communautés religieuses (Azzaoui, 2009: 3), leurs homologues américains se définissent plutôt comme des organisations civiques qui poursuivent des actions profitant à la société dans son ensemble.

Cet engagement avec les normes politiques est également observé dans la discussion de Yükleyen et Tunagür (2013) sur l'influence des différences contextuelles politiques et économiques sur les activités transatlantiques du Mouvement islamique Gülen (aussi connu comme le Mouvement Hizmet). Ce mouvement d'origine turque se concentre sur l'intégration des jeunes Turcs dans les cultures locales en créant des institutions éducatives. Contrairement à ses filiales européennes, ses branches outre-Atlantiques concentrent également leurs activités sur le dialogue interreligieux. Selon les auteurs, cette divergence est expliquée par certains facteurs contextuels locaux, mais aussi par le fait que les membres de Gülen en Europe font généralement partie de la classe ouvrière, alors que la plupart des militants de ce mouvement aux États-Unis proviennent de la classe moyenne, ce qui conduit à des différences dans les attentes internes et les ressources.

Enfin, la recherche de Gest sur les OI $(2010,2013)$ à Londres et à Madrid offre une preuve supplémentaire que la diversité des formes d'organisation est en soi un résultat de structures d'opportunités nationales et locales. Il en examine plusieurs allant des mosquées, aux groupes d'intérêts particuliers, aux associations culturelles. Il affirme que cette variété dans la structure organisationnelle est une adaptation au contexte national lui-même. Les musulmans choisissent certaines formes d'organisation en réponse aux environnements civiques dans lesquels des associations de volontaires sont un moyen 
reconnu pour l'engagement politique, ainsi qu'en réponse à des divisions internes entre les communautés islamiques locales et à l'exclusion croissante des acteurs musulmans des processus politiques en Espagne et au Royaume-Uni. En résumé, l'approche ascendante nous permet de comprendre comment les OI réagissent à des SOP locales spécifiques lorsqu'elles optent pour une forme ou une stratégie organisationnelle particulière.

Facteur contextuel 2: Opportunités discursives. Cette section se concentre sur l'impact des opportunités discursives découlant de débats publics nationaux sur les stratégies des OI. Koomen et Van Heelsum (2013) ont trouvé dans leur recherche comparative sur les OI dans cinq pays d'Europe occidentale que, dans les cinq cas, on peut voir émerger un accent transnational sur les questions ethnoculturelles et religieuses plutôt que sur les questions socio-économiques. Cette différence reflète les débats médiatiques qui identifient les inégalités ethnoculturelles et religieuses comme les obstacles les plus importants à l'intégration. Les acteurs musulmans se concentrent sur les grands enjeux des débats publics dans leurs pays d'établissement, plutôt que sur les questions propres aux musulmans. En outre, des représentants islamiques n'essaient pas seulement de devenir des participants visibles dans les débats sur des questions politiques ayant un impact sur les musulmans et sur les non-musulmans, mais ils doivent également faire des commentaires sur les questions majeures.

Les recherches faites par Malik (2013) démontrent que les organisations musulmanes au Royaume-Uni font face à des pressions similaires pour élargir leur répertoire discursif en abordant la question de l'extrémisme religieux et l'aide aux autorités dans leurs efforts contre le terrorisme, même si elles n'estiment pas qu'il en va de leur responsabilité. Cette expansion du répertoire discursif est une adaptation aux images négatives de l'islam au Royaume-Uni, où les OI sont obligées d'améliorer la réputation de la minorité musulmane, parfois au détriment de problèmes plus locaux. Rosenow-Williams (2012) constate des tendances similaires dans l'analyse du contexte allemand.

Contrairement à d'autres groupes musulmans, les Ahmadis sont confrontés au défi unique d'être une «minorité dans une minorité». Les recherches faites par Beyeler (2013) sur les communautés ahmadies en Suisse ont montré que les stratégies utilisées par les associations musulmanes locales ahmadies pour soutenir leurs projets de construction de mosquée sont fondamentalement façonnées par des discours au niveau de la municipalité sur les musulmans en Suisse. Par exemple, un débat très animé sur un sujet socio-politique dans une municipalité a entraîné la communauté locale ahmadie à utiliser des stratégies plus défensives que la communauté ahmadie d'une autre municipalité où le débat était moins passionné. Cette recherche met en évidence le fait que les Ahmadis doivent se défendre contre certains musulmans sunnites qui pensent que les Ahmadis ne sont pas des musulmans. De plus, ils doivent se défendre contre l'islamophobie des non-musulmans.

Ces études empiriques démontrent que la recherche en perspective ascendante permet aux chercheurs de comprendre (1) comment les OI participent aux discours publics et politiques dans des contextes où les musulmans sont minoritaires et (2) comment ils tentent de concilier leurs propres besoins avec les attentes formulées par leurs environnements organisationnels. 
Facteur contextuel 3 : Les régimes d'incorporation des immigrants et les liens transnationaux. Les différences entre les OI dans les pays européens et aux États-Unis peuvent également être attribuées à des régimes divergents d'intégration des immigrants. Cet argument a été soulevé dans divers volumes ayant une approche descendante sur l'étude de «l'intégration» de l'islam dans les pays à majorité non-musulmane (Kymlicka, 2009; Laurence, 2012). Cet article contribue à examiner la façon dont ces régimes peuvent éclairer l'analyse organisationnelle ascendante. Dans le même temps, l'influence des liens transnationaux et les attentes connectées destinées aux OI à travers les frontières attirent l'attention sur le fait qu'elles sont souvent intégrées dans les régimes d'incorporation de multiples contextes nationaux. Les recherches appliquant une perspective transnationale, posent alors la question de savoir comment les organisations surmontent ce dualisme ou même l'utilisent à leur avantage.

L'influence des régimes d'incorporation des immigrants sur les stratégies des OI est particulièrement apparente dans les recherches de Koomen et Van Heelsum (2013) concernant l'Allemagne. Le régime de nationalité allemande a restreint la naturalisation des immigrés sans ascendance allemande, créant des difficultés pour les immigrants musulmans d'exiger davantage de droits politiques. Kortmann (2014) a aussi trouvé que les définitions de l'intégration adoptées par les organisations turco-islamiques en Allemagne et aux Pays-Bas sont influencées par les régimes d'intégration des immigrants. Bien que les représentants des organisations musulmanes aux Pays-Bas soutiennent une politique d'intégration multiculturelle qui inclut le droit de préserver leur identité d'origine, leurs homologues en Allemagne présentent des formes modérées d'acculturation qui incluent la création d'identités «hybrides» qui combinent des éléments de leur culture d'origine avec la culture de leur pays d'accueil pour se construire comme «musulmans allemands». Cette stratégie est assez similaire au mode dominant d'adaptation chez les musulmans aux États-Unis qui se déclarent comme «musulmans américains », une étiquette qui incorpore des éléments hérités, l'ethnicité, l'islam et l'identité américaine (Sheikh, à paraître).

Loobuyck et Meier (2013) montrent que le travail d'un imam en Flandre implique une charge de travail plus élevée que dans les pays islamiques; ainsi, dans les sociétés à majorité non-musulmane ils doivent conduire la prière, mais doivent aussi surveiller l'éducation religieuse des adultes et des enfants, ils exécutent les mariages et les funérailles, et ils fournissent des conseils aux convertis. De nombreux musulmans vivant dans des pays à majorité non-musulmane ont besoin de conseils plus pratiques sur la façon de vivre leur vie en conformité avec les enseignements de l'islam. Et donc, dans ces contextes, le rôle de conseiller des imams peut être plus important que leur rôle de directeur religieux.

Dans son analyse comparative portant sur l'impact que les liens transnationaux institutionnalisés produisent sur la structure organisationnelle d'un groupe musulman turc en Allemagne (Union turque islamique des affaires religieuses (Diyanet Işleri Türk Islam Birliği, DITIB)) et d'un groupe islamique marocain en France (Rassemblement des musulmans de France, RMF)), Bruce (2013) montre comment ces liens offrent de précieuses ressources à des OI dans les deux nations. L'étude montre que les deux organisations sont en mesure d'obtenir des avantages en utilisant les possibilités - telles que le soutien financier, administratif et politique fourni par les structures institutionnelles, 
y compris les cadres juridiques, les normes et les règles - de leurs pays d'installation, tout en les combinant avec le soutien qu'ils reçoivent de leur pays d'origine.

Une étude sur les Alévis en Allemagne examine également les stratégies que cette minorité islamique utilise dans les domaines multidimensionnels et multi-niveaux dans lesquels elle est impliquée. Les auteurs soutiennent que la «belle réussite de l'alévisme en Europe» (Pries et Tuncer-Zengingül, 2013: 163) est en grande partie due à la capacité des organisations alévies à faire des réclamations, à mobiliser les membres et à influencer les acteurs et les structures politiques dans divers pays européens. En engageant simultanément le champ transnational germano-turc et l'Union Européenne élargie, les alévis allemands maximisent le champ organisationnel dans lequel ils agissent pour les droits des alévis.

En plus de ce rôle de promotion de l'OI, les liens transnationaux ont un impact sur les pratiques (religieuses) dans les mosquées. Beilschmidt (2013) a mené des recherches sur les communautés de mosquées affiliées au DITIB (union des affaires religieuses Turquieislam) en Allemagne, et a constaté que la religiosité parmi les membres de la DITIB est devenue une affaire de plus en plus privée. Cette privatisation croissante est due aux liens transnationaux entre l'organisation de coordination DITIB et la Diyanet, qui sont critiqués dans le discours politique allemand. Par conséquent, les membres des sections locales DITIB en Allemagne affichent «une forme de religion privatisée qui ne signifie pas le renoncement à la participation à des organisations religieuses » (Beilschmidt, 2013: 193). Rosenow-Williams (2012) observe dans son étude du DITIB en Allemagne une séparation entre les informations publiées en turc pour les communautés de la mosquée et les communiqués de presse allemands sur le site internet du DITIB. Les deux études analysent les opportunités et les difficultés qui peuvent être associées à des liens transnationaux en fonction du contexte national et du discours.

\section{Conclusion}

Les nombreuses études évaluées dans cette méta-analyse démontrent collectivement que les environnements organisationnels des OI dans les pays à minorité musulmane fournissent des ressources et imposent des restrictions sur les structures et les objectifs des organisations. Certaines prennent une approche défensive en réaction à des sociétés défavorables aux musulmans, tandis que d'autres reconnaissent et font usage des possibilités discursives fournies par les contextes nationaux, et de plus en plus, par les contextes transnationaux. L'étude de Račius (2013) sur les États baltes montre qu'après les attaques terroristes, les musulmans, en général, s'abstiennent de défendre publiquement une amélioration de leur propre statut en raison de craintes de représailles islamophobes de la part de l'État et de la société. Pries et Tuncer-Zengingül (2013), d'autre part, identifient les alévis en Allemagne comme un groupe islamique qui a été perçu comme «libéral» et «intégré», et qui a réussi à s'auto-défendre pour une reconnaissance officielle en tant que communauté religieuse en Allemagne.

Cette évaluation de la recherche sur les OI révèle que se référer aux contextes du pays d'accueil ou du pays d'origine ne relève plus d'une question exclusive 'soit/soit'. La recherche évaluée dans cette analyse indique que les SOP transnationales permettent aux organisations d'établir et de maintenir des liens dans différents pays et de cultiver les 
identités transnationales (voir, par exemple, le mouvement Gülen, les alevis, ou les mosquées associées au Diyanet turque).

Cependant, les études ont également montré que les SOP peuvent différer considérablement selon les contextes locaux au sein d'une nation. Ce dernier point est particulièrement exact pour les opportunités discursives les plus dynamiques qui ont parfois un impact plus fort que les SOP institutionnels (plus stables) sur la façon dont les OI agissent. Par conséquent, l'élargissement de l'approche des SOP en intégrant les possibilités discursives s'est avéré fructueux. Il en va de même pour la conceptualisation des opportunités découlant de régimes de gouvernance religieuse (voir Fetzer et Soper, 2005), car ils diffèrent à la fois dans les pays européens ainsi qu'entre l'Europe et les États-Unis.

Deux conclusions sur l'impact des différences nationales peuvent être tirées de cette analyse comparative, axée sur les OI. Premièrement, les contextes nationaux influencent plusieurs dimensions de la communauté des OI, ce qui conduit à des formes, des stratégies et des pratiques différentes (Kortmann, 2011). Deuxièmement, les contextes nationaux expliquent seulement quelques développements dans le domaine de l'organisation.

Les intérêts individuels et ceux de groupes musulmans dans des pays à minorité musulmane jouent aussi un rôle important, et ces intérêts évoluent avec le temps. Deux exemples incluent les impacts du statut socio-économique et des différences générationnelles des musulmans d'une nation donnée (Nalborczyk et Ryszewska 2013; Račius 2013 ; Rosenow-Williams, 2012; Yükleyen et Tunagür, 2013). Les membres des OI doivent s'adapter aux besoins variés des groupes musulmans différents dans une société donnée en offrant de nouveaux services au travers des structures organisationnelles actuelles, ou en fondant de nouvelles organisations.

L'hétérogénéité des formes d'organisation est donc un produit de structures et de règles d'opportunité externes, qui expliquent bon nombre des défis rencontrés par les musulmans et leurs organisations, mais aussi la diversité interne des intérêts entre ceux qui s'identifient et s'organisent eux-mêmes comme des musulmans. Cette diversité à l'intérieur de la communauté musulmane n'est pas seulement liée à des facteurs contextuels de la sphère religieuse, mais aussi à des différences de statut social et d'intérêts politiques à l'histoire de l'immigration, aux origines ethniques et géographiques, ainsi qu'à des questions de sexe, d'âge et d'éducation.

\section{Recommandations pour de futures recherches}

L'examen démontre que des approches ascendantes des études des OI aident à détecter et à interpréter leurs formes, stratégies et pratiques, tout en basant sur les idées générées par des études qui ont analysé d'autres «religions immigrantes », particulièrement dans le contexte américain depuis les années 1990 (cf. Warner et Wittner, 1998; Ecklund et Cadge 2007).

Adopter une approche ascendante pour l'étude des musulmans organisés et des diverses formes d'OI nécessite souvent des méthodes qualitatives. Resserrer le contact entre les chercheurs et les membres de la communauté musulmane est une stratégie importante pour des projets de recherche. De futures études à long terme devraient continuer à utiliser cette approche. Employer la triangulation des méthodes qualitatives 
(observation participante, entretiens narratifs) avec des méthodes quantitatives (enquêtes, analyse basée sur le contenu) permettra de fournir de nouvelles perspectives.

Les conclusions des articles analysées dans cette étude révèlent la nature transitoire et remarquable des OI et de leurs relations avec leurs environnements. Comme dans toute société, les processus de transition continuent d'influencer les intérêts des musulmans. Dans les pays où les musulmans sont une minorité, cela a souvent conduit à un processus dans lequel les organisations se sont d'abord préoccupées de la satisfaction des besoins religieux, puis elles ont élargi leur gamme de services au fil du temps jusqu'à exercer finalement des pressions pour la reconnaissance et l'acceptation, non pas en tant que «cette autre religion», mais comme partie intégrante et égale de la société (Vermeulen, 2006).

En raison de trajectoires locales et nationales, les organisations continuent d'être des entités hétérogènes confrontées à une multitude d'attentes internes et externes qui posent des défis à la cohésion de l'organisation dans tous les contextes nationaux. Les manières dont les artefacts religieux, les rites, les rituels et les pratiques des OI seront touchés par ces débats soulèvent une question importante pour de plus amples recherches.

Les recherches actuelles expliquées ici indiquent que l'hétérogénéité des formes et des stratégies des OI est susceptible d'augmenter plutôt que de diminuer. Par conséquent, il est important de reconnaître, d'accepter et de soutenir cette diversité et de renforcer la position des musulmans tant dans la société civile que dans les processus politiques, desquels ont souvent été exclus les OI et les représentants musulmans.

Il est également essentiel de garder à l'esprit que beaucoup de musulmans nés en Europe ou aux États-Unis ne perçoivent pas les pays où leurs parents et grands-parents sont nés comme leurs pays d'origine. Par conséquent, les chercheurs qui étudient les minorités musulmanes et leurs organisations dans les pays ayant une majorité nonmusulmane ne devraient pas, a priori, les désigner comme des «étrangers » qui doivent s'adapter aux attentes de leurs «hôtes». Le but de ces analyses est plutôt d'aider à reconnaître les intérêts particuliers des musulmans et de créer les conditions nécessaires pour qu'ils deviennent des parties intégrantes des sociétés dans lesquelles ils vivent.

\section{Financement}

Aucun soutien financier spécifique émanant d'un organisme de financement public, d'une société commerciale ou du secteur non-marchand n'a été attribué à cette recherche.

\section{Notes}

1. En 2010, il y avaient 43,49 millions de musulmans vivant dans les 50 États-nations associés au continent européen, et 2,77 millions aux États-Unis (Pew Research Center, 2012).

2. Les citations de cet article ont été traduites de l'anglais au français par les auteurs.

\section{Références}

Azzaoui M (2009) Similarities in difference: The challenge of Muslim integration in Germany and the United States. AICGS, Issue Brief 33.

Bader V (2007) The governance of Islam in Europe: The perils of modeling. Journal of Ethnic and Migration Studies 33(6) : 871-886. 
Basch L, Glick Schiller N and Szanton Blanc C (1997) Nations unbound: Transnational projects, postcolonial predicaments, and deterritorialized nation-states. Amsterdam: Gordon and Breach.

Beilschmidt T (2013) Religious practices of DITIB mosque community members: Perspectives from Germany. In: Kortmann M and Rosenow-Williams K (eds) Islamic organizations in Europe and the USA: A multidisciplinary perspective. Basingstoke: Palgrave Macmillan, 186-202.

Beyeler S (2013) Negotiating the visibility of Islam in Switzerland: An ethnographic perspective on the conflicts involved with building projects for mosques. In: Kortmann M and RosenowWilliams $\mathrm{K}$ (eds) Islamic organizations in Europe and the USA: A multidisciplinary perspective. Basingstoke: Palgrave Macmillan, 95-110.

Bruce B (2013) Not quite in, not quite out: Islamic organizations in France and Germany and their ties to their states of origin. In: Kortmann $\mathrm{M}$ and Rosenow-Williams $\mathrm{K}$ (eds) Islamic organizations in Europe and the USA: A multidisciplinary perspective. Basingstoke: Palgrave Macmillan, 129-149.

Cesari J (ed.) (2009) Muslims in the West after 9/11: Religions, politics and law. New York: Routledge.

Duyvené de Wit T and Koopmans R (2005) The integration of ethnic minorities into political culture: The Netherlands, Germany and Great Britain compared. Acta Politica 40: 50-73.

Ecklund E and Cadge W (2007) Immigration and religion. Annual Review of Sociology 33 : 359379.

Fetzer JS and Soper CS (2005) Muslims and the State in Britain, France, and Germany. Cambridge : Cambridge University Press.

Gest J (2010) Apart: Alienated and engaged Muslims in the West. New York: Columbia University Press.

Gest J (2013) Divided or conquered: The challenges of organizational life for Muslims in the UK and Spain. In: Kortmann M and Rosenow-Williams K (eds) Islamic organizations in Europe and the USA: A multidisciplinary perspective. Basingstoke: Palgrave Macmillan, 54-75.

Koenig M (2007) Europeanising the governance of religious diversity? An institutionalist account of Muslim struggles for public recognition. Journal of Ethnic and Migration Studies 32(6): 911-932.

Koomen M and Van Heelsum A (2013) The impact of public debates on Muslim representatives in Western Europe: The agenda-setting function of mass media. In: Kortmann $\mathrm{M}$ and Rosenow-Williams K (eds) Islamic organizations in Europe and the USA: A multidisciplinary perspective. Basingstoke: Palgrave Macmillan, 79-94.

Koopmans R and Statham P (2000) Migration and ethnic relation as a field of political contention: An opportunity structure approach. In: Koopmans R and Statham P (eds) Challenging immigration and ethnic relation politics. Comparative European perspectives. Oxford: Oxford University Press, 13-56.

Koopmans R, Staham P, Giugni M et al. (2005) Contested citizenship: Immigration and cultural diversity in Europe. Minneapolis: University of Minnesota Press.

Kortmann M(2011) Migrantenselbstorganisationen in der Integrationspolitik: Einwandererverbände als Interessenvertreter in Deutschland und den Niederlanden. [Auto-organisation des immigrants dans l'intégration politique: les associations d'immigrants comme groupes d'intérêts en Allemagne et aux Pays-Bas] Münster: Waxmann.

Kortmann M (2014) Asking those concerned: How Muslim migrant organisations define integration. A German-Dutch comparison. Journal of International Migration and Integration, DOI : 10.1007/s12134-014-0387-8, diffusion en ligne avant impression, 10 septembre 2014.

Kortmann M and Rosenow-Williams K (eds) (2013) Islamic organizations in Europe and the USA: A multidisciplinary perspective. Basingstoke: Palgrave Macmillan. 
Kymlicka W (2009) The governance of religious diversity. In: Bramadat P and Koenig M (eds) International migration and the governance of religious diversity. Montréal: McGill-Queen's University Press, 323-334.

Laurence J (2012) The emancipation of Europe's Muslims: The State's role in minority integration. Princeton: Princeton University Press.

Loobuyck P and Meier P (2013) Imams in Flanders, Belgium: Toward the first Flemish imams. In: Kortmann M and Rosenow-Williams K (eds) Islamic organizations in Europe and the USA: A multidisciplinary perspective. Basingstoke: Palgrave Macmillan, 169-185.

Love E (2013) Civil liberties or civil rights? Muslim American advocacy organizations. In: Kortmann M and Rosenow-Williams K (eds) Islamic organizations in Europe and the USA: A multidisciplinary perspective. Basingstoke: Palgrave Macmillan, 37-53.

Malik A (2013) Identities, Islamophobia, and the State: Diverse perspectives and experiences of Muslim civic actors from Islamic organizations in the UK. In: Kortmann M and RosenowWilliams K (eds) Islamic organizations in Europe and the USA: A multidisciplinary perspective. Basingstoke: Palgrave Macmillan, 203-223.

Nalborczyk AS and Ryszewska M (2013) Islamic organizations in Poland: From monopoly to pluralism. In: Kortmann M and Rosenow-Williams K (eds) Islamic organizations in Europe and the USA: A multidisciplinary perspective. Basingstoke: Palgrave Macmillan, 13-36.

Nielsen JS, Akgönül S, Alibašić A et al. (2012) Yearbook of Muslims in Europe, Vol. 4. Leiden: Brill.

Østergaard-Nielsen E (2003) Transnational politics: Turks and Kurds in Germany. New York et Londres: Routledge.

Pew Research Center (PEW) (2012) The global religious landscape: A report on the size and distribution of the world's major religious groups as of 2010. Washington, DC: Pew Research Center. Disponible sur: http://www.pewforum.org/files/2014/01/global-religion-full.pdf (consulté le 20 août 2015).

Pries L and Sezgin Z (eds) (2012) Cross-border migrant organizations in comparative perspective. Basingstoke: Palgrave Macmillan.

Pries L and Tuncer-Zengingül T (2013) Neither omnipotent nor a paper tiger: The federation of the Alevi communities in Germany and the dynamics of its multidimensional area activity. In: Kortmann M and Rosenow-Williams K (eds) Islamic organizations in Europe and the USA: A multidisciplinary perspective. Basingstoke: Palgrave Macmillan, 150-166.

Račius E (2013) Islamic organizations in the Baltic States: Commonalities and differences. In: Kortmann M and Rosenow-Williams K (eds) Islamic organizations in Europe and the USA: A multidisciplinary perspective. Basingstoke: Palgrave Macmillan, 111-128.

Rosenow-Williams K (2012) Organizing Muslims and integrating Islam in Germany: New developments in the 21st century. Leiden: Brill.

Schmitter PC and Streeck W (1999) The organization of business interests: Studying the associative action of business in advances industrial societies. MPIfG Discussion Paper 99/1. Cologne.

Sheikh C (à paraître) Culture or religion? Religious and ethnic identity negotiation among secondgeneration Muslim Americans. Journal of Islamic Law and Culture.

Statham P, Koopmans R, Giugni M et al. (2009) Resilient or adaptable Islam? Ethnicities 5(4): 427-459.

Sunier T and Landman N (2015) Transnational Turkish Islam. Shifting geographies of religious activism and community building in Turkey and Europe. Basingstoke: Palgrave Macmillan.

Vermeulen F (2006) The immigrant organising process: The emergence and persistence of Turkish immigrant organisations in Amsterdam and Berlin and Surinamese organisations in Amsterdam, 1960-2000. Amsterdam: Amsterdam University Press. 
Warner RS and Wittner JG (1998) Gatherings in Diaspora: Religious communities and the New immigration. Philadelphia: Temple University Press.

Yükleyen A and Tunagür F (2013) The Gülen movement in Western Europe and the USA. In: Kortmann M and Rosenow-Williams K (eds) Islamic organizations in Europe and the United States USA: A multidisciplinary perspective. Basingstoke: Palgrave Macmillan, 224-241.

\section{Biographies des auteurs}

Matthias KORTMANN est chercheur et chargé d'enseignement au département de Sciences politiques à la Ludwig-Maximilians-Universität München, Allemagne. Après avoir soutenu sa thèse de doctorat sur les organisations de migrants musulmans, à l'Université de Münster (Allemagne), il a travaillé à l'Institute for Migration and Ethnic Studies de l'Université d'Amsterdam (Pays-Bas), et à la Faculté des Sciences économiques et sociales de l'Université de Potsdam (Allemagne). Adoptant une perspective comparative, sa recherche se focalise sur les organisations islamiques considérées comme groupes d'intérêts, sur la politique et la religion en Europe, sur les organisations religieuses dans la politique sociale et sur les politiques d'immigration et d'intégration.

Adresse : Department of Political Science, Oettingenstr. 67, 80538 Munich, Ludwig-MaximiliansUniversitat Munchen, Allemagne

Email : Matthias.Kortmann@gsi.uni-muenchen.de

Kerstin ROSENOW-WILLIAMS est chercheuse et chargée d'enseignement à l'Institute for International Law of Peace and Armed Conflict (IFHV), Ruhr-Universität Bochum, Allemagne. Après avoir étudié la sociologie à Arcata (CA, USA), Bielefeld et Bamberg (Allemagne), elle a soutenu une thèse de doctorat sur les organisations faîtières musulmanes en Allemagne, à la RuhrUniversität Bochum. Elle enseigne actuellement au sein du Network of Humanitarian Action (NOHA) Joint Master's Programme in International Humanitarian Action et à la Faculté des Sciences sociales de la Ruhr-Universität Bochum. Sa recherche se focalise sur les migrations internationales, les actions humanitaires et la sociologie des organisations.

Adresse : Ruhr-Universitat Bochum, Institute for International Law of Peace and Armed Conflict, Bochumer Fenster, Massenbergstraße 9 B, 44787 Bochum, Germany

Email : Kerstin.rosenow@rub.de 